\title{
Expansion of class-J power amplifiers into inverse mode operation
}

\author{
Youngcheol Park ${ }^{\mathrm{a})}$ \\ Dept. of Electronics Eng., Hankuk University of Foreign Studies \\ Yongin-si, Kyunggi-do 449-791, Republic of Korea \\ a) ycpark@hufs.ac.kr
}

\begin{abstract}
In this paper, inverse mode operation of class-J amplifier is introduced. Performances such as dc power dissipation, ac output power, and drain efficiency of the inverse mode class-J are compared with the ones of regular class-J operation. Because of the reduced overshoot in the drain current, the minimum drain voltage in inverse class-J mode can be lowered, so that the output power and drain efficiency are improved. The analysis shows that better efficiency is achieved as the minimum drain voltage is lowered below the knee voltage. For the verification, class-J and inverse class-J power amplifiers at $900 \mathrm{MHz}$ are implemented. The experimental results show that the efficiency of the inverse class- $J$ amplifier is $66.2 \%$ whereas the conventional class- $J$ operation shows $61.2 \%$. This work is expected to be useful for the design of efficient power amplifiers with reasonable linearity.
\end{abstract}

Keywords: efficiency, linearity, power amplifier

Classification: Microwave and millimeter wave devices, circuits, and systems

\section{References}

[1] S. C. Cripps, RF Power Amplifiers for Wireless Communications Second Edition, Norwood, Artech House, 2006.

[2] F. H. Raab, "Class-F power amplifiers with maximally flat waveforms," IEEE Trans. Microw. Theory Tech., vol. 45, no. 11, pp. 2007-2012, Nov. 1997.

[3] S. C. Cripps, P. J. Tasker, A. L. Clarke, J. Lees, and J. Benedikt, "On the Continuity of High Efficiency Modes in Linear RF Power Amplifiers," IEEE Microw. Compon. Lett., vol. 19, no. 10, pp. 665-667, Oct. 2009.

[4] Y. Woo, Y. Yang, and B. Kim, "Analysis and Experiments for HighEfficiency Class-F and Inverse Class-F Power Amplifiers," IEEE Trans. Microw. Theory Tech., vol. 54, no. 5, pp. 1969-1974, May 2006.

[5] P. Wright, et al., "A Methodology for Realizing High Efficiency Class-J in a Linear and Broadband PA," IEEE Trans. Microw. Theory Tech., vol. 57, no. 12, pp. 3196-3204, Dec. 2009. 


\section{Introduction}

The demand for highly efficient communication devices has greatly increased due to the widespread need for energy saving techniques. Accordingly, much effort has been focused on improving the efficiency of wireless power amplifiers. However, although efficiency enhancement designs such as the classF/E/D and Doherty techniques show good efficiency, they suffer from strong nonlinearity and/or complicated circuit topologies $[1,2]$. So far, harmonicshorts for single-transistor amplifiers such as class-B ones have been suggested for the best efficiency of $78.5 \%$. But transistors with low output capacitances at the drain cannot achieve such short circuits at the low-order harmonics due to the series parasitic components inside the package. As well, clipping from the class-B operation does not provide enough linearity for high peak-to-average-power-ratio (PAPR) signals. Therefore, as an alternative, class-J mode operation was introduced to provide the efficiency of class-B operation with non-zero impedance at high harmonics, along with better linearity $[1,2,3]$. Because of their less-saturated operation, class-J amplifiers can carry relatively high PAPR signals without linearization techniques and also show a better dynamic range. However, the peak current in class-J operation is limited by the knee voltage, which degrades the efficiency $[4,5]$. Therefore, in this paper, inverse class-J operation is suggested and analyzed to improve the efficiency by allowing the minimum voltage to be below the knee voltage.

\section{Performance analysis: Class-J and inverse class-J}

As shown in Fig. 1 (a), class-J mode operation has a half-sinusoidal current waveform, and the voltage waveform is mixed with the fundamental and second harmonic components as follows.

$$
v_{J}(\theta)=V_{D C}(1-\cos \theta)(1-\alpha \sin \theta)
$$

and

$$
i_{J}(\theta)=\left\{\begin{array}{cl}
i_{d p} \cos \theta & \text { for }-\frac{\pi}{2}<\theta<\frac{\pi}{2} \\
0 & \text { otherwise }
\end{array}\right.
$$

where $\alpha(-1 \leq \alpha \leq 1)$ is carefully modified to maintain positive output voltage.

Based on the given waveforms, the other performance parameters are calculated for regular class-J operations [3]:

$$
\begin{gathered}
P_{d c}=\frac{i_{d p}}{\pi} V_{D D} \\
P_{o}=\frac{1}{2} \operatorname{Re}\left[v_{1} i_{1}^{*}\right]=\frac{1}{2} \operatorname{Re}\left[\left(v_{D C}-v_{k}\right)(1+j) \frac{1}{2} i_{d p}\right] \\
=\frac{1}{4} i_{d p}\left(V_{D C}-v_{k}\right) \\
R_{L}=\frac{V_{D C}-v_{k}}{1 / 2 i_{d p}}
\end{gathered}
$$




$$
\begin{aligned}
\eta & =\frac{P_{o}}{P_{D C}}=\frac{\pi}{4} \frac{\left(V_{D C}-v_{k}\right)}{V_{D C}} \\
& =\frac{\pi}{4} \frac{\left(V_{D C}-R_{O N} i_{d p}\right)}{V_{D C}}
\end{aligned}
$$

Equation (6) shows that the maximum efficiency is degraded by the knee voltage, $V_{k}$.

In the inverse mode operation, the voltage waveform is half-sinusoidal and the current is a combination of the fundamental and second harmonic components. This mode of operation allows larger voltage swing so that higher output power and better efficiency are achieved [4]. The related current and voltage waveforms for the inverse class-J operation are as follows.

$$
i_{\text {inv.J }}(\theta)=I_{D C, i n v . J}(1-\cos \theta)(1-\alpha \sin \theta),
$$

and

$$
v_{\text {inv.J }}(\theta)=\left\{\begin{array}{cl}
v_{d p, i n v . J} \cos \theta & \text { for }-\frac{\pi}{2}<\theta<\frac{\pi}{2} \\
0 & \text { otherwise }
\end{array}\right.
$$

where $(-1 \leq \alpha \leq 1)$.

Based on the given waveforms, maximum voltage and current swings are expressed in terms of the bias and the knee voltages. In (7), the peak current, $i_{d p, i n v . J}$ is found when $\theta=5 \pi / 4$,

$$
i_{\text {dp,inv.J }}=i_{\text {inv.J }}\left(\frac{5 \pi}{4}\right)=k I_{D C, i n v . J}
$$

where $k \approx 2.914$.

Thus, when $\alpha$ is assumed to be 1 , the fundamental current is expressed by the following equation.

$$
i_{1, i n v . J}=I_{D C, i n v . J}(1+j 1)=\frac{i_{d p, i n v . J}}{k}(1+j 1) .
$$

Similarly, the fundamental voltage component of this inverse mode operation is a function of the peak and the minimum voltages of the transistor, and can be found from the DC voltage of the inverse mode operation, as shown in Fig. 1 (b).

$$
v_{D C, i n v . J}=\frac{1}{\pi}\left(v_{m a x, i n v . J}-v_{m i n, i n v . J}\right)+v_{m i n, i n v . J}
$$

So,

$$
v_{1, i n v . J}=\frac{1}{2}\left(v_{\text {max }, \text { inv.J }}-v_{\text {min,inv.J }}\right)=\frac{\pi}{2}\left(V_{D C}-v_{\text {min, inv.J }}\right),
$$

The $v_{\text {min,inv.J }}$ is the minimum voltage of the inverse mode operation, which is set below the knee voltage.

The third harmonic impedance of the inverse mode is the reciprocal of the regular class-J's because the waveforms of the voltage and current are interchanged with each other as shown in Fig. 1 , and thus $Z_{3 f_{0}}$ is infinite whereas the $Z_{3 f o}$ of regular class-J amplifiers is zero. In addition, the larger voltage 
swing as well as less DC voltage in the inverse mode generates higher output power than the regular class-J does. Also, additional performance parameters such as the optimum load impedance and output power are calculated as follows.

$$
\begin{gathered}
P_{d c, \text { inv.J }}=V_{D C} \frac{i_{d p, i n v . J}}{k} \\
P_{o, \text { inv.J }}=\frac{1}{2} R e\left[v_{1, i n v . J} i_{1, i n v . J}^{*}\right] \\
=\frac{1}{2} R e\left[\frac{\pi}{2}\left(V_{D C}-v_{m i n, i n v . J}\right) \frac{i_{d p, i n v . J}}{k}(1+j)\right] \\
=\frac{\pi}{4 k}\left(V_{D C}-v_{m i n, i n v . J}\right) i_{d p, i n v . J} \\
R_{L, i n v . J}=\frac{\pi}{4} \frac{V_{D C}-v_{m i n, i n v . J}}{i_{d p, i n v . J} / k}
\end{gathered}
$$

Therefore, the efficiency is as follows.

$$
\begin{aligned}
\eta_{\text {inv.J }} & =\frac{\pi}{4} \frac{\left(V_{D C}-v_{\text {min,inv.J }}\right)}{V_{D C}} \\
& =\frac{\pi}{4} \frac{\left(V_{D C}-R_{O N, \text { inv.J }} i_{d p, i n v . J}\right)}{V_{D C}}
\end{aligned}
$$

For the comparison of the efficiencies of class-J and inverse class-J operation, the condition of equal output powers in (4) and (14) is applied, and the following equation is the result of this condition.

$$
\left(\frac{\pi R_{O N}}{k} i_{d p}\right) \beta^{2}-\left(\frac{\pi}{k} V_{D C}\right) \beta+\left(V_{D C}-R_{O N} i_{d p}\right)=0
$$

where $\beta=i_{d p, i n v . J} / i_{d p}$.

A realizable solution to (17) is when $\beta \approx 0.6$, which means that the peak current of the inverse class-J operation is lower than that of class- $J$ for equal output power. Fig. 1 (c) shows the simulated efficiency curves of class-J and inverse class-J operations as the knee voltage varies between $5 \%$ and $10 \%$ of the DC voltage.

From the previous analysis, the harmonic impedances of inverse mode class-J are calculated as shown in Table I.

Table I. Design Parameters of Class-J and Inv. Class-J amplifiers.

\begin{tabular}{c|c|c}
\hline & Class-J & Inv. Class-J \\
\hline$Z_{f o}$ & $(1+j 1) R_{L}$ & $(1+j 1) R_{L, \text { inv.J }}$ \\
\hline$Z_{2 f o}$ & $-j(3 \pi / 8) R_{L}$ & $-j(16 / 3 \pi) R_{L, \text { inv.J }}$ \\
\hline$Z_{3 f o}$ & 0 & $\infty$ \\
\hline$Z_{3 f o}(n>3)$ & 0 & 0 \\
\hline $\begin{array}{c}\text { Loadline } \\
\text { impedance }\end{array}$ & $R_{L}=11 \Omega$ & $R_{L, \text { inv.J }}=28 \Omega$ \\
\hline
\end{tabular}




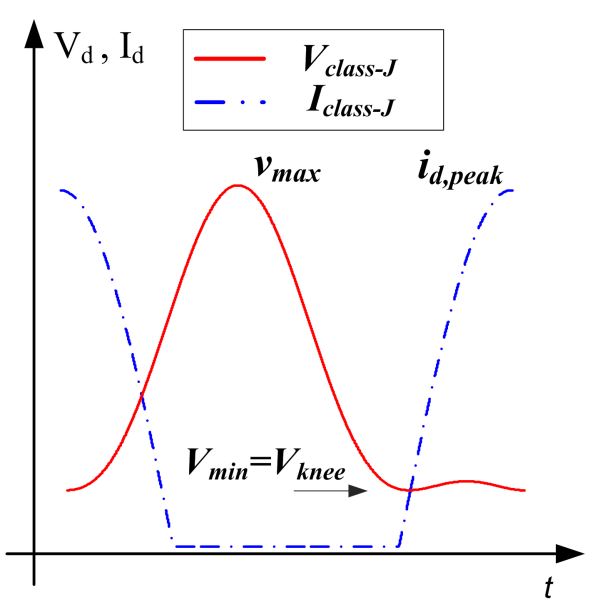

(a)

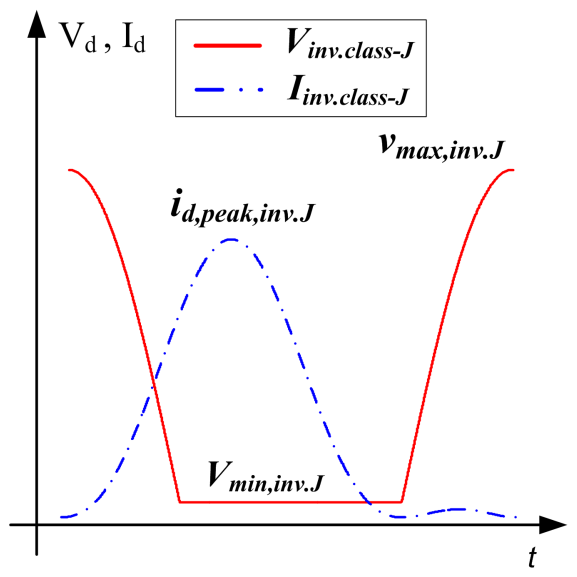

(b)

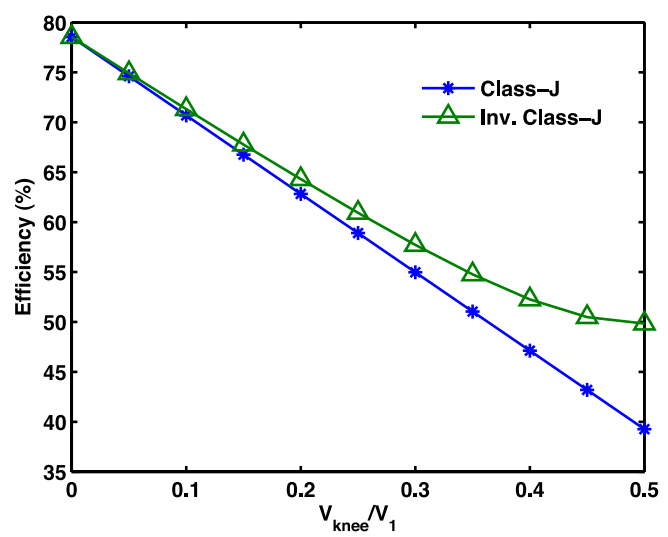

(c)

Fig. 1. (a) Voltage and current waveforms of class-J operation. (b) Voltage and current waveforms of inv. class-J operation. (c) Efficiency curves of class$\mathrm{J}$ and inv. class-J amps. vs. the normalized knee voltage.

\section{Design of an inverse class-J amplifier}

For the verification of the analysis, class- $J$ and inverse class- $J$ amplifiers are designed and simulated at $900 \mathrm{MHz}$ with a commercial transistor, ATF53189 from Agilent Technologies. With $V_{D D}=3 \mathrm{~V}$ and $I_{d, \max }=200 \mathrm{~mA}$, the load impedances and output harmonic impedances are determined based on the analysis of the previous section, and $\alpha$ is adjusted for the optimum performance. Fig. 2 (a) shows the implemented harmonic impedances of the inverse class-J amp. In Fig. 2 (b), output powers of inv. class-J and class-J amplifiers with the input power of 0-10 dBm are compared, indicating higher power is obtained in the inverse mode operation. Simulated and measured performance of the implemented inverse mode class-J amplifier at $900 \mathrm{MHz}$ are shown in Fig. 2 (c), where the peak efficiency reached $66.2 \%$ and stayed above $60 \%$ over $5 \mathrm{~dB}$ of the input power range. For the comparison, the measured peak efficiency of class-J was $61.2 \%$. 


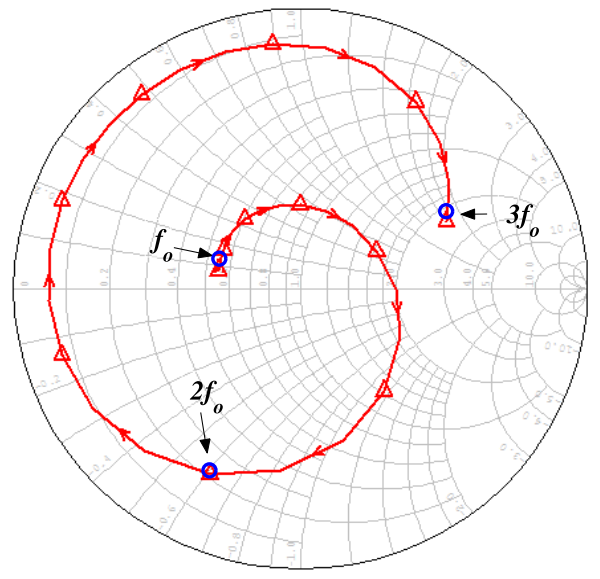

(a)

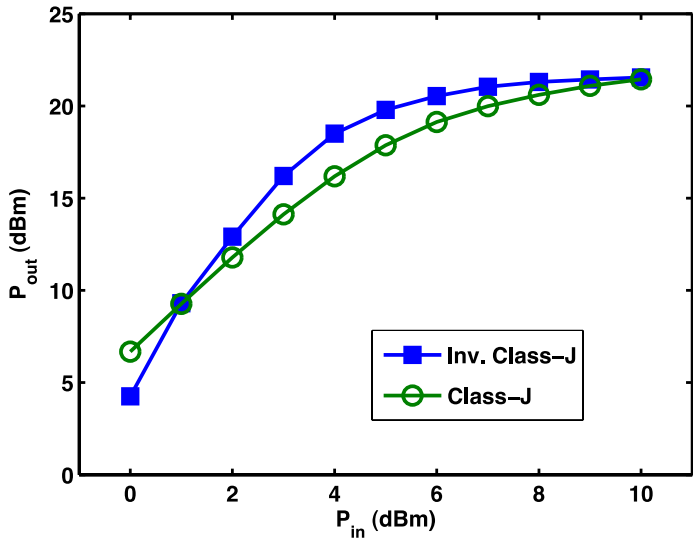

(b)

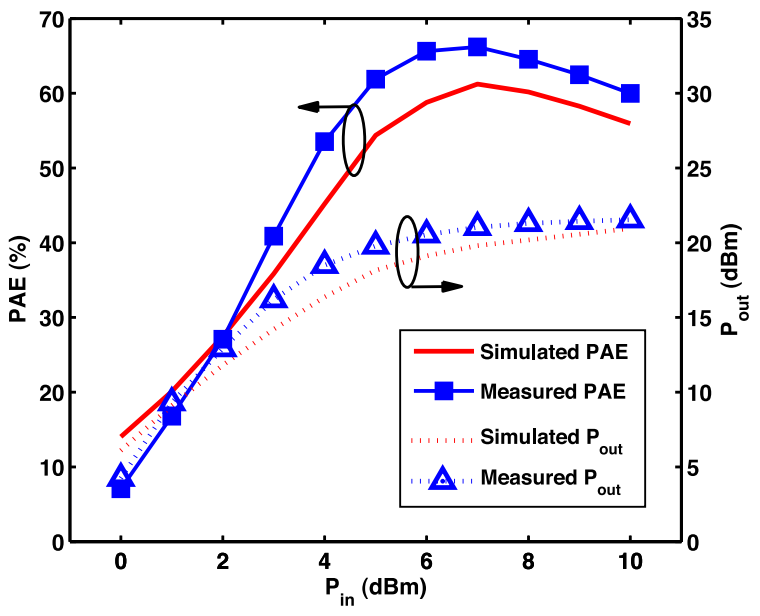

(c)

Fig. 2. (a) Output matching of the implemented inv. class-J amplifier. (b) Comparison of output powers of the inv. and regular class-J amplifiers. (c) Measured efficiency and gain of the inv. class-J amplifier.

\section{Conclusion}

In order to achieve high efficiency over a wide dynamic range and better linearity, class-J amplifiers were introduced. In this paper, to further improve 
the efficiency of class-J operation, the inverse-mode operation of class-J power amplifiers is presented. From the mathematical analysis of the waveform at the drain, it is shown that better efficiency can be achieved in the inverse class-J operation, which is based on the reduction of the overshoot in the drain current. The analysis also shows that the efficiency can be further improved with transistors having higher knee voltages. For the verification, class-J and inverse class-J power amplifiers operating at $900 \mathrm{MHz}$ are designed with commercial pHEMT transistors. The experimental results showed that the efficiency of inverse class-J amplifier was $5 \%$ higher than that of class-J amplifier. This work is expected to be useful for the design of high efficiency power amplifiers with reasonable linearity such as handset power amplifiers, where complex linearization technique is not applicable.

\section{Acknowledgments}

This work was supported by Hankuk University of Foreign Studies Research Fund. of 2011. 